Egyptian

Orthodontic Journal

\title{
DENTO-SKELETAL EFFECTS OF TEUSCHER ACTIVATOR IN TREATMENT OF CLASS II DIVISION 1 MALOCCLUSION CASES
}

\author{
Maher Abd El-Salam Fouda ${ }^{1}$, Ahmad Mohammed Hafez ${ }^{1}$ \\ Mamoun Ali Khawileh²
}

\section{ABSTRACT:}

The purpose of this study was to determine dental and skeletal effects of the of Teuscher activator in treatment of Class II Division 1 malocclusion cases. Sixteen patients (eight males and eight females) with Class Division 1 malocclusion were selected for this study. The mean age of the patients was 10 years 5 months. All patients were treated with the Teuscher activator for one year. Cephalometric radiographs and plaster study models were made at two stages; Gefore and after treatment by the Teuscher activator. The cephalometric radiographs and the study models were analyzed and the collected data were subjected to statistical analysis. Statistical paired $\mathcal{T}$ test was done to determine the significant difference between the pretreatment and posttreatment measurements. Results: The SNA angle was significantly decreased ( $P=.002)$. The SNB angle was significantly increased $(P=000)$. The ANB angle was significantly decreased $(\mathcal{P}=000)$. The mandibular plane, palatal plane, and $\mathcal{Y}$-axis angles $(S \mathcal{N}$ - $\mathcal{M} \mathscr{P}, S \mathcal{N}-\mathcal{P} P$. and $\mathcal{Y}$-axis respectively) were not significantly affected $(\mathcal{P}=.204, \mathcal{P}=.705$, $\mathscr{P}=.041$ and $\mathscr{P}=.064)$. Teuscher functional appliance could be used successfully in treatment of Class II Division 1 malocclusion cases.

1- Professor of Orthodontics, Faculty of Dentistry, Mansoura University.

2- Lecturer of Orthodontics, Faculty of Dentistry, Mansoura University.

3- (B.D.S-)"Nicolae Testemitanu" University (Moldova2003) 
Egyptian

Orthodontic Journal

\section{INTRODUCTION}

Class II malocclusion is characterized by incorrect relationship between the maxillary and mandibular arches because of skeletal problems, dental problems, or a combination of both. Angle Class II malocclusions are considered the most common type of malocclusions. Class II division 1 malocclusion is reported to constitute $12 \%$ to $49 \%$ of all orthodontic disorders and usually causes esthetic and functional problems of varying severity, depending on the amount of anteroposterior discrepancy and its interaction with adjacent soft tissues. Class II malocclusion represents about $21 \%$ of Egyptians especially Class II division $1 .{ }^{1-2-3}$ Most Class II patients have a deficiency in the anteroposterior position of the mandible. Several treatment options are available for managing Class II problems. Class II malocclusions can be treated with many appliances and treatment protocols according to the characteristics of the problem, such as anteroposterior discrepancy, age, and patient compliance. Functional appliances have been used for many years in the treatment of Class II division 1 malocclusions.4- ${ }^{5-6-7-8}$ Two distinct treatment approaches are available for the treatment of Class II division 1 malocclusions: a reduction in maxillary protrusion or an increase in mandibular prominence. A headgear appliance typically achieves reduction of the maxillary prominence. The forward positioning of the mandible is accomplished by using a functional appliance such as the bionator, activator, Fränkel or Twin-block appliance. ${ }^{9-10-11}$ Removable functional appliances and headgears are capable of producing highly successful orthopedic results when worn properly. However, they are completely dependent upon patient cooperation to achieve the desired result. ${ }^{12}$ The primary aim of the present study is to gain substantive information efficacy of Teuscher activator functional appliance on growing patients.

\section{MATERIALS AND METHODS}

Sample: The sample of this study was consisted of sixteen patients (eight males and eight females).The mean age of the patients was 10 years 5 months. The patients were selected from the clinic of Orthodontic Department, Faculty of Dentistry Mansoura University according to the following criteria:

Volume 38-December 2010 
- Dental Class II Division 1 with deep overbite.

- Skeletal Class II due to maxillary protrusion and mandibular retrusion.

- ANB more than $4^{\circ}$.

- Overjet more than $7 \mathrm{~mm}$.

- $\mathrm{SN}-\mathrm{MP}>32^{\circ}$.

- No previous orthodontic treatment.

- No abnormal oral habits.

All patients were treated with Teuscher activator functional appliance for one year.

\section{Records:}

For all patients the following diagnostic records were taken before fitting the appliance.

- Photographs:

A. Intraoral:

1. Frontal view.

2. Lateralviews (Right and Left).

B. Extraoral:

1. Frontal view.

2. Lateralviews.

- Radiographs:

A. Lateral cephalometric radiograph.

B. Panoramic X-ray film.

C. Left hand wrist X-ray film.

- Upper and lower orthodontic casts mounted with wax bite.

All records were taken before and after one year of fitting the Teuscher activator functional appliance except for the hand wrist and panoramic x-ray films which were taken only before treatment. 
Egyptian

Orthodontic Journal

\section{Lateral Cephalometric radiographs:}

Lateral Cephalometric radiographs were taken before and after the treatment and tracings were performed on fine grain 0.003 inch transparent acetate papers using a $0.5 \mathrm{~mm}$ hard lead pencil. Then the following cephalometric points (landmarks), lines and planes were determined.

\section{Cephalometric Points:}

- A Point (Subspinale): The deepest point on the curve of the maxilla between the anterior nasal spine and the dental alveolus.

- B point (Supramentale): The deepest point on the anterior curve of the mandibular symphysis.

- N point (Nasion): A point at the anterior limit of the nasofrontal suture.

-S (Sella): The center of cellaturcicadetermind by vision.

- Me (Menton): The most caudal point in the outline of the symphysis;

- Gn (Gnathion): The most anteroinferior point on the lateral shadow of the chin.

- Go (Gonion): The intersection of the lines tangent to the posterior margin of the ascending ramus and the mandibular base.

- ANS (Anterior nasal spine): The tip of the anterior nasal spine, in the median plane.

- PNS point (posterior nasal spine): The most posterior point on the bony hard plate in the sagittal plane.

- Ui: The tip of the upper incisor.

- Li: The tip of the lower incisor.

- L6M (molar inferius): The distal contact of the mandibular permanent first molar determined by a tangent perpendicular to OL; where double projection gave rise to two points, the midpoint was used.

- U6M (molar superius): The distal contact of the maxillary permanent first molar determined by a tangent perpendicular to OL; where a double projection gave rise to two points, the midpoint was used. 


\section{Cephalometric lines and planes:}

- MP (mandibular plane): It extended from Me to Go.

- PP (palatal plane): It extended from ANS to PNS.

-N-Me: Anterior facial height.

-S-Go: Posterior facial height.

- SN (sella-nasion line): The line through $\mathrm{N}$ and $\mathrm{S}$.

- OL (occlusal plane): A line through incision superius (is) and the distobuccal cusp of the maxillary permanent first molar.

- OLp (occlusal line perpendiculare): A line perpendicular to OL through S. The line from the initial head film was used as reference line for measurements on all head films.

\section{Measuring procedures:}

\section{Linear measeurments:}

- U6M / OLP: Distance from distal crown surface of upper first molar to nasion-sella perpendicular line.

- L6M / OLP: Distance from distal crown surface of lower first molar to nasion-sella perpendicular line.

- OLP /UI: Distance from upper incisor tip to nasion-sella perpendicular line.

- OLP /LI:Distance from lower incisor tip to nasion-sella perpendicular line.

\section{Angular measurements:}

-SNA:Anterioposterior position of the maxilla relative to the anterior cranial base.

-SNB:Anterioposterior position of the mandible relative to the anterior cranial base.

- ANB: Difference between SNA and SNB. 
- MP-SN: Angle between SN and mandibular plane. It givesthe inclination of the mandible to the anterior cranial base.

- PP-SN: Angle between SN and palatal plane.

-UI-SN: The posterior angle formed by extendingthe long axis of the upper incisor to intersect the SN line.

- LI-MP: The posterior angle between the long axis of the lower central incisor and mandibular plane.

- UI-L1 (inter-incisal angle): The angle between long axis of the maxillary and mandibular incisors.

-Y-axis:Sella-nasion to gnathion angle.

\section{Upper and Lower Casts:}

Upper and lower alginate impressions were taken for each patient. The impressions were pured immediately in plaster to produce study casts. Then the study casts were duplicated to produce working plaster models. Via wax bite, the working plaster models were mounted on mean value articulator with the incisal post toughing the incisal table. A digital Boly gauge graduated to the nearest $0.1 \mathrm{~mm}$ was used to perform the following measurements:

I. The intermolar width: The distances between the mesiobuccal cusp tips of the first permanent molars.

II. The intercanine width: The distance between the cusp tips of the cuspids (the estimated cuspids tips in cases of wear facets).

III. Overjet: The horizontal distance from the incisal edge of the most protruded maxillary incisor to that of the opposing mandibular central incisor tooth.

IV. Overbite: The vertical overlap of the crowns of lower incisors by the crowns of upper incisors.

\section{Teuscher Activator Design:}

The Teuscher activator was designed with torquing spurs on the upper incisors fabricated of $0.5 \mathrm{~mm}$ resilient stainless steel.Thetorquing 
Egyptian

Orthodontic Journal

springs were inserted in the acrylic and were in contact only with the upper incisors in one point shortly underneath the gingival margin. It was bent away slightly to avoid contact with the rest of the clinical crown. The retention was in the interocclusal area. The acrylic base was extended laterally to the buccal cusps. In the canine and incisor regions about $2 \mathrm{~mm}$ were covered on the vestibular surface of the crowns. On the lingual side about $3.5 \mathrm{~mm}$ of acrylic support was provided for the incisor crowns. In the buccal segment from the mesial of the canine, the acrylic was extended toward the palatal vault. Instead of covering the entire palate across and forward up to the incisors, palatal bar was replaced the acrylic in a diameter of $1.2 \mathrm{~mm}$. Adequate relief in the acrylic was provided for the lingual frenum. The headgear tubes were inserted in the acrylic between both tooth rows in the area of the deciduous molars or in the premolar area. The interocclusal area was high enough to allow a good anchorage for the retention of the headgear tubes in the acrylic. They also were checked from the occlusal point of view if they were parallel to be able to insert the inner bow without any problems.

\section{Appliance delivery and patient's instructions:}

The fitting of the appliance was checked in each arch of the patient separately then both together when the jaws were closed. The patient was instructed to place the appliance first on the upper arch; otherwise torquing springs would be distorted. If difficulties arose, the undercuts were relieved. The wire elements were not activated initially. High pull face bow was fitted to the activator tubes. The outer arms of the face bow were extended far enough posteriorly in such a way that their ends were corresponded in the frontal plane to the distal cusps of the first permanent molars. The outer arm of the face bow was adjusted and hooked vertically to the head cap. The force applied was $500 \mathrm{gm}$ per side which was graduated by force gauge. The patients were instructed to wear the appliance for the first 3 days 2 hours in the daytime only and from then on also during sleep. After adaptation to the appliance the patient was instructed to wear it 14-hours/day. The first check was after three weeks. Activation of the wire was started in which visit if needed with a light pressure. The check was every 3 weeks. At every visit, the fit of the appliance was checked and the progress of the treatment was assessed. If 
eruption of the lower permanent teeth was started, the acrylic was removed occlusally. If curve of spee needed to be leveled, acrylic was removed occlusal to the lower premolars. If the lower arch length was appearing to be critical, the first molar position was secured by spur mesial to it. The acrylic was extended distally to the second molar (if present) to prevent its overeruption.

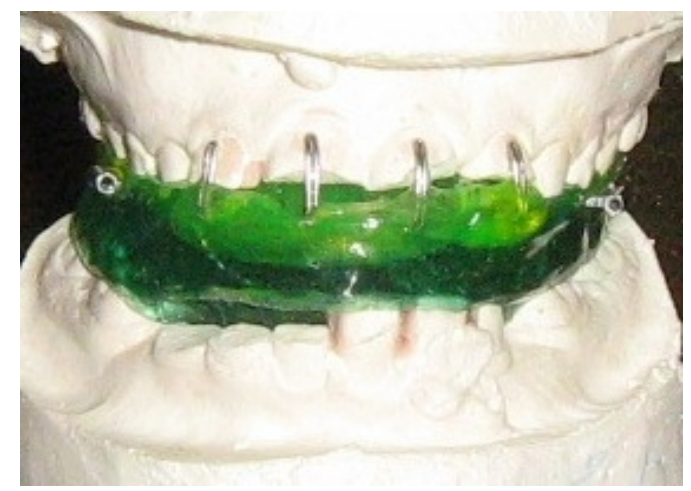

(Figure 1) Teuscher activator.

\section{Statistical Analysis:}

All measurements were calculated and analyzed using the SPSS statistical program. Descriptive statistics including means and standard deviations of the measurements before and after treatment were obtained. Then, $\mathrm{t}$ - paired test was done to test the significance difference between the pretreatment and posttreatment measurements (Table I and Table II).

\section{RESULTS}

The period of treatment was one year. The skeletal measurements changes were significant for the sample before and after treatment. The maxillary forward growth was restricted as the SNA angle which was significantly decreased $(\mathrm{P}=.002)$. The mandibular forward growth was stimulated as the SNB angle was significantly increased $(\mathrm{P}=000)$. Finally, the maxillomandibular relationship was improved. The ANB angle was significantly decreased $(\mathrm{P}=000)$. The mandibular plane, palatal plane, and $\mathrm{Y}$-axis angles (SN-MP, SN-PP. and Y-axis respectively) were not significantly affected $(\mathrm{P}=.204, \mathrm{P}=.705, \mathrm{P}=.041$ and $\mathrm{P}=.064)$. On other 
hands, the Occlusal plane angle $(\mathrm{OL} / \mathrm{SN})$ was significantly affected $(\mathrm{p}=.041)$. The anterior and posterior facial heights (N-ME and S-GO respectively) were not significantly affected $(\mathrm{P}=.227$ and $\mathrm{P}=.250)$. The overjet was significantly decreased $(\mathrm{P}=.000)$. The upper incisors were significantly retroclined and moved palatally as UI/ OLP distance and UI/SN angle were significantly dcreased $(\mathrm{P}=.013 \& \mathrm{P}=.028$ respectively $)$. The lower incisors were significantly proclined as LI/ OLP distance and LI/ME-GO angle were significantly increased $(\mathrm{P}=.051 \& \mathrm{P}=.016$ respectively).The $\mathrm{UI} / \mathrm{LI}$ angle was significantly increased $(\mathrm{P}=.001)$ The mandibular first molar showed a significant mesial movement in the L6/ OLP distance $(\mathrm{P}=.012)$. On the other hand, the maxillary first molar showed a significant distal movement (i.e.) there was a decrease in the U6/ OLP distance $(\mathrm{P}=.004)$. There was a significant correction of the overjet $(\mathrm{P}=.000)$. The overbite showed a significant decrease $(\mathrm{P}=.000)$. The lower intercanine width and the upper intercanine width showed a nonsignificant change $(\mathrm{P}=.50 \& \mathrm{P}=.057$ respectively). Also the upper and lower intermolar widths showed a nonsignificant change $(\mathrm{P}=.209 \& \mathrm{P}=.050$ respectively).

Table I: The means, standard deviations (SD), and the results of t-test of the pretreatment and posttreatment cephalometric measurements.

\begin{tabular}{|l|c|c|c|c|}
\hline \multirow{2}{*}{ Measurements } & Prereatment & Posttreatment & \multirow{2}{*}{ t } & \multirow{2}{*}{ p-value } \\
\cline { 2 - 3 } SNA (degree) & Mean \pm SD & Mean \pm SD & & $.002^{*}$ \\
\hline SNB (degree) & $83 . \pm 2.002$ & $81.05 \pm 1.49$ & 4.48 & $.000^{*}$ \\
\hline ANB (degree) & $74.6 \pm 2.22$ & $75.6 \pm 2.06$ & -6.70 & $.000^{*}$ \\
\hline SN-MP (degree) & $38.7 \pm 1.15$ & $5.45 \pm 1.83$ & 7.14 & .204 \\
\hline SN-PP (degree) & $11.20 \pm 1.39$ & $11.40 \pm 1.83$ & -.39 & .705 \\
\hline Y-axis (degree) & $68.70 \pm 3.19$ & $70.00 \pm 3.018$ & -2.11 & .064 \\
\hline SN -OL (degree) & $21.70 \pm 1.88$ & $23.30 \pm 2.47$ & -2.38 & $.041^{*}$ \\
\hline N-ME (mm) & $119.0 \pm 7.408$ & $121.20 \pm 8.91$ & -1.29 & .227 \\
\hline S-GO (mm) & $75.80 \pm 8.85$ & $77.70 \pm 10.44$ & -1.23 & .250 \\
\hline
\end{tabular}

Significant: $\mathrm{P}<0.05^{*}$

Volume 38 - December 2010 
Table II: The means, standard deviations (SD), and the results of t-test of the pretreatment and posttreatment of dental measurements.

\begin{tabular}{|l|c|c|c|c|}
\hline \multirow{2}{*}{\multicolumn{1}{|c|}{ Measurements }} & Prereatment & Posttreatment & \multirow{2}{*}{ t } & \multirow{2}{*}{ p-value } \\
\cline { 2 - 3 } & Mean \pm SD & Mean \pm SD & & \\
\hline UI/ OLP $(\mathrm{mm})$ & $74.10 \pm 8.49$ & $68.70 \pm 8.26$ & 3.07 & $.013^{*}$ \\
\hline LI/ OLP $(\mathrm{mm})$ & $62.20 \pm 6.62$ & $65.40 \pm 5.29$ & -2.25 & .051 \\
\hline UI/SN(degree) & $107.20 \pm 6.49$ & $104.10 \pm 6.88$ & 2.61 & $.028^{*}$ \\
\hline LI/ME-GO(degree) & $100.40 \pm 5.66$ & $104.20 \pm 6.88$ & -2.96 & $.016^{*}$ \\
\hline UI/LI(degree) & $118.40 \pm 6.11$ & $123.40 \pm 4.81$ & -4.84 & $.001^{*}$ \\
\hline U6/OLP(mm) & $24.50 \pm 5.48$ & $20.90 \pm 4.43$ & 3.80 & $.004^{*}$ \\
\hline L6/ OLP (mm) & $20.60 \pm 4.22$ & $22.50 \pm 5.25$ & -3.14 & $.012^{*}$ \\
\hline overjet $(\mathrm{mm})$ & $8.00 \pm 1.054$ & $3.40 \pm .61$ & 15.057 & $.000^{*}$ \\
\hline overbite $(\mathrm{mm})$ & $4.30 \pm .67$ & $2.90 \pm .39$ & 7.79 & $.000^{*}$ \\
\hline UICW $(\mathrm{mm})$ & $35.25 \pm 2.58$ & $35.38 \pm 2.52$ & -2.17 & .057 \\
\hline LICW(mm) & $27.83 \pm 2.89$ & $27.97 \pm 2.84$ & -2.24 & .050 \\
\hline UIMW $(\mathrm{mm})$ & $45.40 \pm 2.91$ & $45.47 \pm 2.94$ & -1.35 & .209 \\
\hline LIMW(mm) & $41.05 \pm 2.11$ & $42.20 \pm 1.68$ & -2.26 & .050 \\
\hline
\end{tabular}

Significant: $\mathrm{P}<0.05^{*}$

\section{DISCUSSION}

The Class II, Division 1 malocclusion is the most frequent treatmentproblem in the orthodontic practice. The treatment can involve the use of functional or fixed orthodontic appliances, or both. The purpose of the present study was to evaluate the Teuscheractivator functional appliance. The sample was made with special criteria. It was done with respect to basic factors which could influence the treatment results. These factors included; sex, ethnic background, skeleto-facial morphology, and growth status. The study included male and female patients. All patients were growing and had the same ethnic background, and with high angle skeletal Class II malooclusion. In the present study, lateral cephalometric x-ray films and study casts were taken before and after one year of treatment with Teuscheractivator functional appliance. The cephalometric analysis was done according to Martin and Pancherz (2009). 
Egyptian

Orthodontic Journal

\section{Skeletal effects}

\section{Maxilla}

The Teuscher activator functional appliance caused a significant inhibition of maxillary growth of the maxilla especially in the horizontal direction. A significant decrease in the SNA angle was found. This result was in agreement with other functional appliances studies of Pauw and Dermaut $^{13}$, Levin ${ }^{14}$, Toutountzakis and Sifakakis ${ }^{15}$ and Nassar and Abdelnaby ${ }^{16}$.

\section{Mandible}

The Teuscher activator functional appliance stimulated the mandibular growth. In the present study, a significant increase in the SNB angle was found. This result was in agreement with functional appliance studies of Pauw and Dermaut ${ }^{13}$, Levin ${ }^{14}$, Toutountzakis and Sifakakis ${ }^{15}$, Nassar and Abdelnaby ${ }^{16}$.

\section{Maxillomandibular relationship}

In the present study, maxillomandibular relationship was improved as the ANB angle was significantly decreased. This result was in agreement with those of Pauw and Dermaut ${ }^{13}$, Levin ${ }^{14}$, Toutountzakis and Sifakakis ${ }^{15}$ and Nassar and Abdelnaby ${ }^{16}$,

\section{Vertical dimension}

Rrgarding the vertical dimension, the use of Tuscher functional appliance led to increas in the posterior facial height (S-Go) and the anterior facial height $(\mathrm{N}-\mathrm{Me})$ but the changes were not significant. This result was in agreement with other functional appliances studies of Levin $^{15}$. On the other hand, it was in contrast to the results of Nassar and Abdelnaby ${ }^{16}$. On the other hands, OL-SN angle was decreased but not significantly.

\section{Dental effects}

\section{Incisors}

Several researchers reported that combined activator headgear treatment resulted in a significant increase in lower facialheight, as Pauw 
and Dermaut ${ }^{13}$, Levin ${ }^{14}$, and Nassar and Abdelnaby ${ }^{16}$. This is in line with the results of the current study; the upper incisors showed a noticeable palatal movement and retroclination as UI- NSP distance and the UI- SN angle decreased significantly. Rrgarding the lower incisor position, there was a proclination related to (OLP) distance and (ME-GO) angle were increased significantly. This result was in agreement with other functional appliances studies of Pauw and Dermaut ${ }^{13}$, Levin ${ }^{14}$, Nassar and Abdelnaby 16.The UI/LI angle were increased significantly. The overjet and overbite were decreased significantly. These findings were in accordance with those of Pauw and Dermaut ${ }^{14}$, Levin ${ }^{15}$, an Nassar and Abdelnaby ${ }^{16}$, The dental changes were recorded as retroclination of the upper incisors and proclination of lower incisors.

\section{Molars}

The maxillary first molars showed significant distal movement as U6/OLP distance was decreased significantly. And this was in agreement with Nassar and Abdelnaby ${ }^{16}$ Pauw and Dermaut ${ }^{13}$, Levin ${ }^{14}$, Regarding the position of the lower molars, a significant mesial movement was showed as the L6/ OLP distance was. The upper and lower molar relationship was improved. The U6/ OLP and L6/ OLP distances were significantly changed. The U6/ OLP distance was decreased while the L6/ OLP distance was increased. This results were in agreement with other functional appliances studies of Pauw and Dermaut ${ }^{13}$, Levin ${ }^{14}$, Nassar and Abdelnaby ${ }^{16}$, The upper intermolar, lower intermolar and lower intercanine widths (UIMW, LIMW, UICW and LICW) were nonsignificant increased.

\section{CONCLUSIONS}

From the present study the followings could be concluded:

1) Teuscher functional appliance was effective in management of Class II Division 1 via skeletal and dental effects.

2) The use of Teuscher functional appliance may achieved dental, and skeletal changes in the sagittal plane, by restraining the maxillary growth potential and advancing the mandible, ensuing improvement of the profile.

3) Teuscher functional appliance did not change the vertical dimension of the face. 

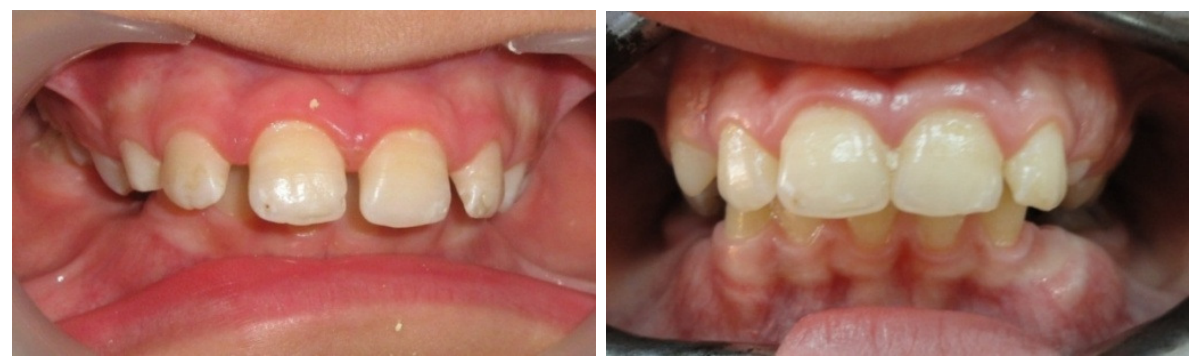

(Figure 2) (intraoral photograph Left: pretreatment; right: Posttreatment)

\section{REFERENCES}

1. Bishara S E 1998 Mandibular changes in persons with untreated and treated Class II division 1 malocclusion. American Journal of Orthodontics and Dentofacial Orthopedics $113: 661-673$.

2. de Oliveira JN Jr, Rodrigues de Almeida R, Rodrigues de Almeida M, de Oliveira JN: Dentoskeletal changes induced by the Jasper jumper and cervical headgear appliances followed by fixed orthodontic treatment. Am J Orthod Dentofacial Orthop. 2007 Jul; 132(1):54-62.

3. Fouda, M.A.: Disturbance of the accepted occlusion by extraction. Al Azhar D.J 1987;2 (5): 453-67.

4. Cançado RH, Pinzan A, Janson G, Henriques JF, Neves LS, Canuto CE: Occlusal outcomes and efficiency of 1- and 2-phase protocols in the treatment of Class II Division 1 malocclusion. Am J Orthod Dentofacial Orthop. 2008 Feb; 133(2):245-53.

5. Sidlauskas A, Svalkauskiene V, Sidlauskas M: Assessment of Skeletal and Dental Pattern of Class II Division 1 Malocclusion with Relevance to Clinical Practice. Stomatologija. 2006; 8(1):3-8.

6. Bass NM: Orthopedic coordination of dentofacial development in skeletal Class II malocclusion in conjunction with edgewise therapy. Part I. Am J Orthod 1983;84:361-83.

7. Casutt C, Pancherz H, Gawora M, Ruf S.: Success rate and efficiency of activator treatment.. Eur J Orthod. 2007 Dec;29(6):614-21. Epub 2007 Sep 18. 
8. O'Brien KD, Robbins R, Vig KWL, Vig PS, Shnorhokian H, Weyant R. The effectiveness of Class II Division 1 treatment. Am J Orthod Dentofac Orthop 1995;107:329-34.

9. Keeling SD, Wheeler TT, King GJ, Garvan CW, Cohen DA, Cabassa S, McGorray SP, Taylor MG. Anteroposterior skeletal and dental changes after early Class II treatment with bionators and headgear. Am J Orthod Dentofacial Orthop. 1998 Jan;113(1):40-50.

10. Singh GD, Clark WJ: Localization of mandibular changes in patients with Class II Division 1 malocclusions treated with Twin-block appliances.Finite element scaling analysis. Am J Orthod Dentofacial Orthop 2001; 119:419-25.

11. Dugoni SA, Lee JS, Varela J, Dugoni AA. Early mixed dentition treatment: postretention evaluation of stability and relapse. Angle Orthod 1995;65:311-20.

12. Sargiss G, Elkin B, Epstein J, Kapoor S, Morgenstern T, Epstein M: Dentofacial Orthopedics.The Herbst Appliance. brace place. 2009, January, 4, 1Page 3.

13. Pauw and Dermaut: A Modified Teuscher Activator. Semin Orthod 2006;12:34.

14. Levin R.L: Activator headgear therapy: Am J Orthod Dentofacial Orthop. 1985 Feb (91-109)

15. Toutountzakis N, Sifakakis I: Combination of activator and extraoral forces. Case presentation and literature review. Hellenic Orthodontic Review 2006; 9:123-141.

16. Nassar. E. A and Abd-Elnaby. Y. A: Dento-Skeletal effects of Twin Block-Headgear in treatment of Class IImalocclusion cases. EOJ. 2009 December; 36. 